\title{
Individuation et identité chez Diderot
}

\author{
MITIA RIOUX-BEAULNE Université d'Ottawa
}

RÉSUMÉ : Cet article s'attache à un aspect fondamental de la philosophie matérialiste de Diderot, à savoir le fait que l'individualité psychologique ne peut pas correspondre à l'individu matériel que nous sommes parce que la mémoire sur laquelle elle repose est toujours en quelque sorte partielle. Que faire alors si cet individu matériel lui-même voit son existence mise en doute, du fait que, comme l'annonce le Rêve de d'Alembert, le seul individu, c'est le «tout»? En mettant en relation ces deux démarches, nous montrons comment on peut élaborer une théorie de l'individu qui tienne compte du décalage entre l'individuation comme processus physique et de la constitution de l'individualité comme processus psychologique.

ABSTRACT: This paper is devoted to a fundamental aspect of Diderot's materialist philosophy: the fact that psychological identity does not exactly correspond with the material individuals we are because the memories on which psychological identity relies are always partial. This is complicated by the fact that even the material individual is denied reality since, as is stated in the Rêve de d'Alembert, the only individual is the "whole». By relating these two approaches, I try to show how one can develop a theory of the individual that takes into account the gap between individuation as a physical process and the constitution of a psychological individual.

Mots-clés : Denis Diderot, individuation, identité, métaphysique, philosophie moderne

\section{Introduction}

33 Il sera ici question d'un décalage. Parler d'individuation et d'identité comme 34 de lieux théoriques distincts semble signifier, d'entrée de jeu, qu'il n'est pas 35 évident que ce que ces mots désignent puisse jamais concorder. Il est vrai que, 36 dans les métaphysiques de l'âge classique, ces lieux semblent généralement 37 être traités comme des problèmes distincts. D'une part, en effet, le concept

Dialogue 0 (2017), 1-28.

(C) Canadian Philosophical Association/Association canadienne de philosophie 2017 doi:10.1017/S0012217317000713 
1 d'individuation problématise la formation des corps, lesquels, en dépit de leur 2 divisibilité et des changements constants qui les affectent, semblent bien 3 répondre à un principe unificateur qui nous permet de les traiter comme des phénomènes ayant une unité propre. D'autre part, celui d'identité vise l'instance d'un «moi» qui, par-delà l'indivisibilité de l'idée que nous en avons, doit pouvoir accueillir une variété d'états. Ainsi, le problème est démultiplié : la vérité du corps est sa divisibilité, mais il faut expliquer son unité apparente; la vérité du moi est son indivisibilité, et il faut expliquer la diversification incessante de ses manifestations.

Les grandes philosophies de la période moderne ont donné diverses solutions à ce problème, allant tantôt dans le sens d'une affirmation de la substantialité du moi comme chose pensante opposée à ce que l'on pourrait qualifier d'unité purement phénoménale du corps - dont Descartes est le paradigme tantôt dans le sens d'un scepticisme qui tend à «désubstantialiser» le moi et le corps auquel il semble uni - et ici, c'est Hume sans doute que l'on peut invoquer comme figure exemplaire. Une troisième stratégie demeure possible, mais elle a moins fait l'objet d'études approfondies : une stratégie matérialiste, si l'on veut, au sens où elle pose la substantialité du corps individué et déréalise l'apparente unité du moi.

Dans ce qui suit, il s'agira poser un certain nombre de jalons pour une telle stratégie, en utilisant comme point d'ancrage la pensée de Denis Diderot ${ }^{1}$. La façon dont Diderot aborde le problème est en effet intéressante, parce qu'elle reconnaît la pertinence de poser le problème dans les termes des adversaires du matérialisme tout en en disqualifiant les conclusions. Ainsi, comme on le verra, Diderot s'efforce de trouver une explication de l'individuation qui réponde à deux écueils traditionnels : la dilution du concept de corps individué dans une théorie de la matière comme totalité homogène ou sa substantialisation outrancière tendant à l'isoler de la totalité. Par ailleurs, Diderot s'efforce de produire une conception de l'identité psychologique qui évite les apories d'un substantialisme de la chose pensante dont l'interaction avec les choses matérielles apparaît incompréhensible, sans pour autant en faire un pur épiphénomène dont on pourrait tout aussi bien faire l'économie. À terme, donc, il s'agit de montrer que le moi désigne une fonction du corps humain par laquelle cet organisme parvient à saisir dans la forme de l'identité à soi ce qui constitue sa vie à titre de forme individuée transitoire.

Notons d'emblée que les problèmes de l'identité et de l'individuation ont déjà fait l'objet d'un certain nombre de travaux au sein des études diderotiennes depuis quelques années, suivant en cela un mouvement général des études modernistes. Ces études ont réussi progressivement à faire admettre et

1 Ce choix se justifie par le fait que Diderot est, parmi les philosophes matérialistes d'Ancien Régime, celui qui s'est peut-être le plus attardé aux problèmes canoniques de la métaphysique traditionnelle. À ce sujet, voir Bourdin (1998). 
comprendre la richesse de la proposition diderotienne quant à la formation du sujet sous les auspices d'une conscience de soi qui soit un effet du corps ${ }^{2}$. De plus, les travaux portant sur les aspects physiologiques de la pensée de Diderot ont permis de mieux comprendre comment celui-ci mobilise différents savoirs pour asseoir une pensée de l'individuation corporelle sur une conceptualité scientifique à laquelle il donne une extension philosophique maximale ${ }^{3}$, souvent par des jeux de métaphorisation revendiqués à titre de moyens d'anticiper sur des savoirs empiriques à venir ${ }^{4}$. Ce qui ressort de ces études, c'est que le matérialisme diderotien constitue une tentative de réponse authentiquement philosophique aux problèmes hérités des métaphysiques du XVII ${ }^{\mathrm{e}}$ siècle ${ }^{5}$. En ce qui concerne les questions de l'identité personnelle et de l'individuation corporelle, l'intérêt philosophique de la pensée de Diderot apparaît précisément tenir au fait qu'elle les traite comme les deux faces d'une même question. C'est précisément sur cette dimension de l'approche diderotienne que je voudrais mettre ici l'accent, en vue d'en montrer la fécondité6.

2 Trois études classiques s'attachent à ce problème : Bourdin (1999), Salaün (1999) et Duflo (2008). Soulignons aussi, à ce titre, Wolfe (2011), qui aborde la pensée de Diderot, ainsi que trois ouvrages entièrement consacrés à ces questions et qui ouvrent sur elles des perspectives particulières : Cammagre (2000), Jacot-Grapa (2009) et Salaün (2010).

3 Une étude fondatrice, à notre sens, sur cette question, demeure celle de François Duchesneau (1999). Pour des développements encore plus récents, on consultera la troisième partie de Pépin (2012, p. 667-726).

4 Catherine Glyn Davies (1990) a montré à ce titre que Diderot participe à une tendance générale du siècle des Lumières à revaloriser le raisonnement par analogie. En ce qui a trait à Diderot plus particulièrement, elle identifie six groupes de métaphores récurrentes qui servent à aborder la question de l'identité personnelle. Voir les pages 108-152 de son ouvrage pour une analyse détaillée de ces métaphores. On lira aussi, sur la même question, Dion-Sigoda (1992).

5 Voir, sur ce point, mes deux contributions récentes : Rioux-Beaulne $(2015,2016)$.

6 Je vais centrer mon étude sur un groupe de textes datant de la période 1769-1773 où ces questions sont centrales. Le rêve de d'Alembert (1769) met en scène sous la forme d'un dialogue en trois parties ce qui constitue sans doute la forme la plus achevée du matérialisme de Diderot, en abordant les problèmes traditionnels de la métaphysique. Pour cette raison, je le mettrai au cœur de mes analyses. J'y joindrai quelques références à d'autres textes, notamment à ce qu'il est convenu aujourd'hui d'appeler les Observations sur Hemsterhuis (1773) : il s'agit en fait de notes prises dans les marges de la Lettre sur l'homme du philosophe hollandais, qui ont l'insigne intérêt de n'avoir pas été écrites dans l'horizon d'une publication, et conséquemment d'exprimer souvent de manière très directe certaines des conséquences du matérialisme que Diderot veut défendre. 


\section{Individuation}

La question de l'individuation est sujette, chez Diderot, à deux types de formulation, renvoyant chacune à un héritage philosophique avec lequel il cherche à composer.

Le premier type relève de l'épistémologie : il s'agit de déterminer le degré de réalité que l'on peut accorder aux entités qui apparaissent phénoménologiquement comme des corps individués, séparés les uns des autres. En définissant les corps comme des modes d'une substance unique, Descartes avait mis la table pour une critique de l'ordre des phénomènes. Les sens donnent à voir un monde de corps séparés par des vides là où il n'y a qu'une substance diversement arrangée. Ainsi, il n'y a pas de vide, mais une substance raréfiée :

Nous disons qu'un espace est vide lorsqu'il ne contient rien qui nous soit sensible, encore qu'il contienne une matière créée et une substance étendue. Car nous ne considérons ordinairement les corps qui sont proches de nous, qu'en tant qu'ils causent dans les organes de nos sens des impressions si fortes, que nous pouvons les sentir ${ }^{7}$.

Suivant ce schéma, l'ordre des phénomènes se présente comme un espace vide habité par des corps séparés les uns des autres parce que les parties de matière, lorsqu'elles sont raréfiées comme dans le cas de l'air, n'offrent pas une résistance suffisante pour modifier les organes de nos sens; et l'âme, ne percevant aucune modification du corps auquel elle est unie, prend pour des espaces vides ceux qui sont faits de telles parties de matière raréfiées. Elle est alors conduite à traiter les corps qui y évoluent comme s'ils étaient des substances distinctes, plutôt que des modes d'une substance unique.

Le second type de formulation est lié au développement de la philosophie corpusculaire et à ses conséquences ontologiques. Il s'agit cette fois, la réalité de la matière étant posée - fût-ce à titre de simple hypothèse —, de déterminer ce qui autorise le traitement d'une partie de matière comme un corps individué.

Reprenons, ici aussi, les Principes de la philosophie comme point de départ. Descartes y décrit le processus d'individuation des corps à partir du mouvement local :

Par un corps, ou bien par une partie de la matière, j'entends tout ce qui est transporté ensemble, quoiqu'il soit peut-être composé de plusieurs parties qui emploient cependant leur agitation à faire d'autres mouvements (Descartes, Principes, II, 25, p. 76).

Commentant ce passage, Alice Sowaal (2004) fait remarquer que la difficulté posée par la physique cartésienne tient au fait que le mouvement local d'un

7 René Descartes, Principes de la philosophie, II, 17, dans Euvres, sous la direction d'Adam et Tourneux, vol. IXb (voir Descartes, 1897-1913, p. 72). Dorénavant désigné par l'abréviation Descartes, Principes, [partie], [section], [page] dans le corps du texte. 
corps, c'est-à-dire ce par quoi il apparaît avoir une unité propre qui lui procure une existence séparée de ce qui l'environne, relève du simple déplacement simultané de ses parties. En conséquence, il est permis de penser que Descartes ne pose nulle part de réalité ontologique des corps séparés - l'individuation pourrait n'être, en définitive, que phénoménale. Dans un univers plein, fait d'une substance matérielle unique, seules les variations du mouvement local sont réputées être responsables de la diversité des phénomènes. Sans entrer ici plus avant dans les débats sur la théorie cartésienne de l'individuation des corps, ces remarques visent simplement à rappeler que pour une ontologie qui refuse d'admettre la réalité du vide et qui veut faire l'économie des formes substantielles, les corps semblent voués à n'avoir d'autre réalité qu'idéale.

Évidemment, ce que nous présentons ici comme deux formulations distinctes du problème de l'individuation ne constitue en fait que les deux faces d'une même médaille. Il s'agit, pour chaque formulation, d'aborder la question sous un angle différent. Dans le premier cas, l'enjeu est la légitimité des jugements portés sur la correspondance de l'ordre des phénomènes avec celui des substances : peut-on arracher une portion de substance au tout auquel elle appartient pour la traiter comme une entité ayant une réalité ontologique propre (et si oui, peut-on le faire sur la base de ce qui est perçu par nous comme distinct)? Dans le second cas, le problème est celui des propriétés que l'on confère à la substance, ce qui permet de traiter un groupe de corpuscules se mouvant ensemble comme une seule instance faisant corps autrement que de manière purement phénoménale. Autrement dit, dans le premier cas, partant de la perception, on demande s'il est légitime de traiter le corps individué comme une entité séparée; dans le second, partant d'une notion de la substance, s'il est légitime de traiter des entités séparées comme un seul corps individué. Or, si l'on est condamné à aborder la question sur ces deux axes à la fois, c'est d'une certaine manière - Diderot lui-même le souligne que l'idée que nous nous faisons de la matière est en elle-même problématique.

Presque toutes les formes de la matière sont indivisibles. Il n'y a pas la moitié d'un doigt, la moitié d'un œil; il n'y a pas la moitié d'une molécule quelconque, parce que l'une de ces moitiés occupant une autre place que l'autre moitié, par cette seule et unique raison, elle ne lui ressemble pas. Cependant, la matière est divisible Voilà donc deux modifications contradictoires, selon vous, réunies dans un même sujet ${ }^{8}$.

Le matérialisme de Diderot s'offre comme une voie afin d'essayer de sortir de l'impasse.

8 Denis Diderot, Observations sur Hemsterhuis, DPV, XXIV, p. 297. DPV est l'abréviation désignant l'édition des Euvres de Diderot chez Hermann commencée en 1975 par H. Dieckmann, J. Proust et J. Varloot (33 volumes prévus). Les références à cette édition sont dorénavant désignées dans le corps du texte par l'abréviation Diderot, [Titre de l'œuvre], DPV, [volume], [page]. 


\subsection{Un seul individu}

Diderot souligne en plusieurs endroits de son œuvre qu'une ontologie matérialiste cohérente doit accepter de mettre de côté la notion d'individu pour désigner une partie de la nature, si par individu on veut signifier une réalité autonome ${ }^{9}$. Certes, Diderot reconnaît qu'il doit y avoir des parties élémentaires de la nature molécules, atomes - qui sont indivisibles ${ }^{10}$. Toutefois, l'indivisibilité ne confère à ces éléments aucune individualité au sens philosophique du terme ${ }^{11}$ — ce ne sont 8 pas des monades ou des atomes qui entretiendraient une relation d'identité à soi 9 stable et permanente ${ }^{12}$. Pris dans le cours du devenir, l'être de chacun de ces

Toute chose est plus ou moins une chose quelconque, plus ou moins terre, plus ou moins eau, plus ou moins air, plus ou moins feu; plus ou moins d'un règne ou d'un autre... donc rien n'est de l'essence d'un être particulier... Non, sans doute, puisqu'il n'y a aucune qualité dont aucun être ne soit participant... et que c'est le rapport plus ou moins grand de cette qualité qui nous la fait attribuer à un être exclusivement à un autre... Et vous parlez d'individus, pauvres philosophes! laissez là vos individus : répondez-moi. Y a-t-il un atome en nature rigoureusement semblable à un autre atome?... Non... Ne convenez-vous pas que tout tient en nature et qu'il est impossible qu'il y ait un vide dans la chaine? Que voulez-vous donc dire avec vos individus? Il n'y en a point, non, il n'y en a point... Il n'y a qu'un seul grand individu, c'est le tout (Diderot, Le rêve de d'Alembert, DPV, XVII, p. 138-139).

L'insistance à considérer que le seul individu est le tout sert ici avant tout, on le voit, une thèse de type phénoméniste : il s'agit de montrer qu'il faut déréaliser ce

9 Diderot, Pensées sur l'interprétation de la nature, par. XI, DPV, IX, p. 35 : «L'étonnement vient souvent de ce qu'on suppose plusieurs prodiges où il n'y en a qu'un; de ce qu'on imagine dans la nature autant d'actes particuliers qu'on nombre de phénomènes, tandis qu'elle n'a peut-être jamais produit qu'un seul acte... L'indépendance absolue d'un seul fait est incompatible avec l'idée de tout.»

10 Diderot, Le rêve de d'Alembert, DPV, XVII, p. 97 : «La divisibilité de la matière a un terme dans la nature, quoiqu'elle n'en ait point dans l'entendement.»

11 À titre de référence, l'Encyclopédie (VIII, p. 864) donne cette définition : «INDIVIDU, s. m. (Métaphysiq.) : c'est un être dont toutes les déterminations sont exprimées». Nous citons l'Encyclopédie à partir de l'édition originale à Paris, chez Durand et al., 17511765 (27 volumes), en employant dorénavant la forme Encyclopédie, [volume], [page].

12 «Il n'y a donc pas de stabilité de l'identité individuelle, même au niveau des molécules élémentaires, mais au contraire un flux perpétuel à tous les niveaux de la vie et de la sensibilité. Le problème de la notion d'individu est là : Le rêve de d'Alembert détruit progressivement la possibilité d'unités fermées sur elles-mêmes, identiques à elles-mêmes... Quel sens peut alors conserver la notion d'individu?» demande Sophie Audidière (2006, p. 231). 
qui apparaît comme séparé dans l'ordre des phénomènes et le rabattre sur un continuum. Traiter les corps matériels comme des individus relève d'une conception erronée de la nature :

Dans ce tout, comme dans une machine, dans un animal quelconque, il y a une partie que vous appellerez telle ou telle; mais quand vous donnerez le nom d'individu à cette partie du tout, c'est par un concept aussi faux que si, dans un oiseau, vous donniez le nom d'individu à l'aile, à une plume de l'aile... Et vous parlez d'essences, pauvres philosophes! Laissez là vos essences. Voyez la masse générale (Diderot, Le rêve de d'Alembert, DPV, XVII, p. 139).

La formule est brutale, mais elle fait bien voir ce qui est en cause : les corps individués sont en fait des parties d'un tout; par un «concept faux», nous substantialisons ces parties du tout, ce qui nous amène à les traiter comme des choses dont on peut penser l'existence séparée. L'aile, la plume de l'aile ne sont pourtant pas des individus même si nous en avons des concepts distincts - or, ce qui est vrai de l'aile par rapport à l'oiseau l'est aussi bien de l'oiseau par rapport au tout de la nature. Ce n'est que par une sorte de réification de l'ordre phénoménal que ces parties sont détachées du système des relations qui les unissent au tout. De fait, l'ordre des phénomènes est au fond conditionné par le type et le degré de sensibilité des organes par lesquels le monde se donne à nous. Diderot juge que la structure du monde phénoménal est en fait dépendante du mode d'être de celui qui perçoit.

Bernard Baertschi (2005, p. 73-100) a bien montré comment, dans les objections qu'il fait à Condillac dès la Lettre sur les aveugles de 1749, Diderot retient l'interprétation cartésienne de la sensation comme modification de l'organe perceptif, lui adjoint la thèse empiriste voulant que cette sensation est le seul accès à l'être que nous ayons, et vient ainsi ébranler l'idée qu'il puisse y avoir correspondance entre l'ordre des phénomènes et celui des choses. Condillac, suivi en cela par nombre d'empiristes français, a été amené à substituer l'impénétrabilité à l'extension comme propriété la plus fondamentale des corps et, conséquemment, à accepter l'existence du vide comme un réquisit de l'intelligibilité scientifique du monde ${ }^{13}$.

13 Pour Condillac, à partir du Traité des sensations de 1754, cela apparait dans le privilège accordé, dans la deuxième partie du livre, au toucher comme sens permettant de penser l'extériorité de la cause des sensations. Mais la meilleure illustration vient peut-être de l'article Vuide (anonyme) de l'Encyclopédie, lequel affirme : «Puisque l'essence de la matière ne consiste pas dans l'étendue, mais dans la solidité ou dans l'impénétrabilité; on peut dire que l'univers est composé de corps solides qui se meuvent dans le vide : \& nous ne devons craindre en aucune manière que les phénomènes, qui s'expliquent dans le système du plein, se refusent au système de ceux qui admettent le vuide» (XVII, p. 573). D'Alembert affirme également que l'impénétrabilité est une propriété commune à tous les corps dans l'article CoRps de l'Encyclopédie (IV, p. 262). 


\section{Dialogue}

1 Ce faisant, une sorte de passage à la limite s'opère : une propriété phénoménale 2 sert à établir une propriété ontologique ${ }^{14}$. Rappeler que les individus sont des 3 «concepts faux» a donc dans un premier temps pour fonction de mettre en cause ces tentatives de fonder cette espèce de réalisme strict ${ }^{15}$.

\subsection{Il n'y a pas un seul être}

Cet argument est lié la nécessité d'instaurer un cadre épistémique ne s'appuyant plus sur l'ordre des phénomènes ${ }^{16}$. Ce faisant, le matérialisme apparaît plus plausible à titre d'ontologie générale, puisqu'il repose sur l'idée d'une

14 B. Baertschi (2005, p. 81 et sq.) parle à ce sujet d'un mouvement de «double attribution» associé au toucher, lequel provoque une sorte d'instabilité dans la pensée primauté du tout de la nature. Une fois posé ce principe, il est en revanche nécessaire de fournir de la matière un concept qui, fut-ce de manière provisoire, ait un degré de cohérence et de fécondité explicatives suffisant à rendre compte des phénomènes ${ }^{17}$. de Condillac, oscillant entre phénoménisme obligé par la définition de la sensation comme modification de l'âme et réalisme visé par un effort de fondation sur le privilège du toucher comme mode de découverte d'une propriété fondamentale des corps.

15 C. Jacot-Grapa remarque que «l'aporie du toucher semble dire que l'attouchement, ou le "serré" comme l'appelle Diderot dans la Lettre sur les aveugles, n'est pas un plus pur gage de vérité que la vision» (2009, p. 361).

16 Ceci n'est évidemment pas sans rappeler une critique similaire chez Spinoza (1954, Partie I, proposition 8, scolie 2, p. 24-25), qui rappelle que la difficulté de saisir qu'il n'y a qu'une substance et qu'elle est infinie tient à la confusion que nous faisons entre substances et modifications.

17 C'est là, en effet, le sens de ce que Diderot avait développé dans le cadre de l'article Spinosiste de l'Encyclopédie : «SPINOSISTE, s.m. (Gram.) sectateur de la philosophie de Spinoza. Il ne faut pas confondre les Spinosistes anciens avec les Spinosistes modernes. Le principe général de ceux-ci, c'est que la matière est sensible, ce qu'ils démontrent par le développement de l'œuf, corps inerte, qui par le seul instrument de la chaleur graduée passe à l'état d'être sentant \& vivant, \& par l'accroissement de tout animal qui dans son principe n'est qu'un point, \& qui par l'assimilation nutritive des plantes, en un mot, de toutes les substances qui servent à la nutrition, devient un grand corps sentant et vivant dans un grand espace. De-là ils concluent qu'il n'y a que de la matière, et qu'elle suffit pour tout expliquer; du reste ils suivent l'ancien spinosisme dans toutes ses conséquences» (XV, p. 474 - nous soulignons la dernière phrase; pour le passage sur l'œuf, nous ne l'avons pas tronqué puisqu'il permet de voir par anticipation ce que nous allons défendre dans cette section). Nous n'entrerons pas ici dans le détail d'une justification de cette suffisance explicative. Disons seulement, à la suite de Jean-Claude Bourdin (1998, p. 74 et sq.), que les trois critères épistémologiques fondamentaux qui gouvernent la pratique 
Ce qui rend l'individuation des corps problématique dans la philosophie cartésienne, c'est le fait de les avoir définis comme des ensembles de parties de matière se mouvant ensemble, sans que ces parties ne semblent entretenir de relation spécifique entre elles. Elles apparaissent liées entre elles à l'être qui les perçoit parce qu'elles se meuvent ensemble, et elles apparaissent déliées du reste parce que ce reste ne se meut pas de concert avec elles - ce qui nous a permis d'affirmer dans la section précédente que l'individuation des corps chez Descartes pourrait n'être en définitive que phénoménale. Cela tient à une suite de principes, dont trois ici sont de toute première importance :

a) La matière est définie comme substance divisible indéfiniment (Descartes, Principes, II, 34, AT, IXb, p. 82);

b) Toutes les modifications qui l'affectent sont réductibles au mouvement local - ce qui en fait une substance homogène (Descartes, Principes, II, 23, AT, IXb, p. 75);

c) Le mouvement a été imprimé à la matière par un agent extérieur - en l'occurrence, Dieu (Descartes, Principes, II, 36, AT, IXb, p. 83).

En ce qui concerne le troisième principe, nombre de philosophes du XVIII siècle adoptent la stratégie globalement newtonienne qui consiste à considérer la formation des corps à partir de deux règles : 1) on peut prêter des propriétés dynamiques à la matière comme le fait de renfermer une force d'attraction ${ }^{18}$, et 2) la formation des corps s'explique par la cohésion de parties de matière qui résulte du jeu de ces propriétés dynamiques ${ }^{19}$. Cette posture comporte l'avantage de distinguer entre ce qui peut passer pour un corps alors qu'il ne s'agit que de parties de matière se mouvant ensemble (ex. : un nuage) et un corps au sens propre en tant qu'il s'agit de parties de matière effectivement liées les unes aux autres par une force cohésive (ex. : une table). Si ce modèle permet de rendre compte des variations de dureté et d'élasticité des corps, il demeure

philosophique de Diderot sont la correspondance entre les attendus d'une hypothèse et l'expérience, la cohérence interne et la fécondité d'une explication. Voir également Colas Duflo (2006, p. 182), qui écrit : «Il suffit que l'hypothèse de la matérialité intégrale du réel convienne pour interpréter intégralement l'ensemble des effets qu'on observe dans la nature, pour qu'on soit légitimé à l'adopter. Si le monisme matérialiste explique tout, et mieux que les hypothèses concurrentes, c'est-à-dire d'une façon à la fois plus rationnelle, plus économique et plus complète, alors il est justifié»».

18 Voir par exemple l'article Corps de l'Encyclopédie (IV, p. 262-263), signé par d'Alembert.

19 Voir l'article Attraction de l'Encyclopédie (I, p. 847), également de d'Alembert. Pour des détails sur ces questions, voir Firode (2001), Gandt (2001) et Pépin (2012, p. 590 et sq.). 
1 lacunaire sur un point qui est, aux yeux de Diderot, de la plus haute importance : 2 c'est qu'il ne parvient pas à fournir un critère de distinction entre les corps qui 3 sont l'effet d'une agglomération (ex. : une table), et ceux dont la cohésion est,

On doit comprendre ici que la perspective à partir de laquelle ceci est énoncé est précisément celle qui définit l'individuation à partir de la seule cohésion mécanique de parties de matière. Or, dans un passage qui suit immédiatement celui-ci, Diderot poursuit :

L'homme qui prendrait cette grappe pour un animal se tromperait [...]. Voulez-vous transformer la grappe d'abeilles en un seul et unique animal? Amollissez les pattes par lesquelles elles se tiennent, de contiguës qu'elles étaient rendez-les continues. Entre ce nouvel état de la grappe et le précédent il y a certainement une différence marquée; et quelle ne peut être cette différence sinon qu'à présent c'est un tout, un animal un, et qu'auparavant ce n'était qu'un assemblage d'animaux (Diderot, Le rêve de d'Alembert, DPV, XVII, p. 120-121).

L'importance de cette distinction vient de ce que Diderot juge l'ajout de l'attraction aux propriétés de la matière insuffisante à l'explication de ce qui est en cause, à savoir le fait que certains corps - les corps animaux — constituent des agrégats dont le comportement ne s'explique pas par la seule cohésion mécanique de parties de matière. C'est que ces corps, contrairement à ceux qui ne sont que le produit d'une cohésion par attraction, forment «un animal un», une entité dont il est légitime de dire qu'elle a une sorte d'individualité - et ce, en dépit de son inévitable liaison au tout dont elle est une partie.

Le refus de Diderot de faire de la cohésion mécanique le seul principe d'individuation est issu d'une critique plus générale à l'égard d'une conception 
uniquement physico-mathématique de l'attraction. Cette critique porte sur le fait qu'une telle définition restreinte de l'attraction renvoie au second principe que nous avons évoqué plus haut, à savoir que la matière est une substance homogène dont toutes les modifications se comprennent comme des effets du mouvement local. La difficulté est, on le sait, liée au fait que dans un tel monde, le mouvement aurait inévitablement tendance à décliner s'il n'était maintenu par un agent extérieur ${ }^{20}$. En ce sens, le seul ajout de l'attraction aux propriétés de la matière telle que définie par Descartes demeurerait insuffisant à exclure tout recours à l'action divine pour expliquer le mouvement incessant qui affecte la matière et qui assure non seulement la formation de corps par cohésion de parties de matière, mais la variété des rapports que les parties de matière peuvent entretenir. C'est en décrivant la matière comme hétérogène que Diderot parvient en quelque sorte à sortir de cette impasse. L'hétérogénéité des parties de la matière fournit, dans un premier temps, un argument pour la perpétuation du mouvement sans recourir à un agent externe :

L'ordre général varie sans cesse. Tout est in fluxu et eterno et perpetuo et necessario.

Celui qui nous frappe actuellement, et que nous admirons parce que nous coexistons supportablement avec lui, passera; et quand le tout se réduira-t-il à une masse; je l'ignore. Vraisemblablement, jamais... Il n'y a qu'un seul cas pour la masse et la masse inerte; c'est celui où toutes les parties homogènes, hétérogènes seraient distribuées les unes entre les autres, de manière à constituer partout une parfaite unité, un seul être (Diderot, Observations sur Hemsterhuis, §167, DPV, XXIV, p. 317).

L'idée qu'il n'y ait «qu'un seul cas» où tout se résoudrait en une grande masse inerte signifie que l'hétérogénéité de la matière rend improbable l'agencement de la matière en une seule masse inerte (Diderot, Observations sur Hemsterhuis, \$173, DPV, XXIV, p. 321). L'hétérogénéité de la matière transforme le cadre conceptuel qui sert à penser les relations entre les parties de matière. Au-delà des relations mécaniques (mouvement, choc, agglomération...), on peut désormais envisager des passages de la contiguïté à la continuité, des effets de répulsion, etc., de sorte qu'il puisse y avoir formation de masses partielles qui ne se définissent plus par le seul fait de se mouvoir ensemble, ou d'être des agrégats :

Il est démontré géométriquement et de plusieurs manières, que sans aucune force d'impulsion, quelconque, de trois corps homogènes, placés dans le vide, et animés par la seule force d'attraction, il y a une infinité de cas où l'un des trois décrira une ellipse autour des deux autres.

Ôtez la loi d'homogénéité, multipliez la somme des forces ou actions.

20 On se souviendra que c'était déjà l'une des objections principales de Leibniz à la physique newtonienne : voir Maizeaux (1720, p. 4). 
Rendez celle des corps infinie.

Et jugez de ce que cela deviendra.

Quelle multitude de masses partielles et diverses!

$[\ldots]$

Il y a des philosophes qui ont trouvé assez de fécondité pour suffire à tous les phénomènes dans le mouvement et la divisibilité d'une matière homogène. Mais ils se trompaient. Combien n'auraient-ils pas été plus à leur aise si à ces deux principes ils avaient ajouté l'attraction, les affinités et les cinq éléments divers (Diderot, Observations sur Hemsterhuis, §169-170, DPV, XXIV, p. 318-319)21.

On voit, par ce qui précède, que Diderot cherche dans l'hypothèse de l'hétérogénéité de la matière une ressource permettant de surmonter ce qu'il considère être les apories tant des cartésiens que d'un certain type de newtonianisme. Une telle hypothèse s'autorise, selon Diderot, des ressources conceptuelles de la chimie, science qui rend possible en effet d'introduire à l'intérieur du concept de matière une panoplie de propriétés qui débordent largement ce que permet le cadre épistémique des sciences physico-mathématiques en s'appuyant sur une démarche expérimentale. Diderot parvient ainsi à produire un concept général de matière ${ }^{22}$ qui, en se soustrayant aux deux principes que nous avons évoqués plus haut (extériorité de l'origine du mouvement et homogénéité de la matière), rend possible une théorie de l'individuation des corps fondée sur la pluralité des relations qu'occasionne l'hétérogénéité des parties de la matière. Conséquemment, Diderot parvient à former une solution «immanentiste» à la question de l'individuation qui, au lieu d'être ancrée dans l'individualité fixe et originaire d'éléments, de corpuscules homogènes donnée une fois pour toute, se donne la relation comme fondement : certaines parties de matière ont des relations telles qu'elles constituent des individus tant que durent ces relations - et inversement, loin d'être attachée à une essence

21 Suivant l'éditeur de ce texte, la première partie prolonge un développement des Pensées sur l'interprétation de la nature (DPV, IX, p. 60-62), où Diderot visait déjà une preuve newtonienne de l'existence de Dieu, et dont il perçoit des échos dans le texte d'Hemsterhuis qu'il commente.

22 On ne peut que suggérer, pour des approfondissements sur cette question, de consulter Pépin (2012, p. 595 et sq.). Par exemple, Pépin donne du concept de matière chez Diderot la définition suivante : «nom donné à l'ensemble des matières hétérogènes, concept nominal et relationnel qui ne recoupe aucun être individuel réel (au sens où un corps n'est jamais de la matière, mais telle matière). Ce nom regroupe conformément au nominalisme sans essence de Diderot un ensemble de propriétés et permet de penser leurs rapports dynamiques» (ibid., p. 608). 
1 propre, l'individualité d'une partie de matière est un effet des relations qu'elle 2 entretient avec le reste ${ }^{23}$.

On parvient alors à ce qui, dans cette approche, permet enfin de contester le premier des trois principes cartésiens que nous avons présentés - celui qui concerne la divisibilité de la matière. En effet, s'il est vrai, comme nous l'avons montré dans la partie précédente, qu'il n'y a pas d'autre individu que le tout, il est également vrai que ce tout ne constitue pas un plan homogène, mais plutôt un flux perpétuel donnant lieu à des agencements formant des masses partielles, des êtres à part entière et distincts les uns des autres, agissant les uns sur les autres. Dans les termes de Diderot, certaines parties de matière sont capables de passer d'un état de contiguïté à un état de continuité, autrement dit d'un «assemblage d'animaux» à un «animal un» ${ }^{24}$.

Toutes les fois que cet état de continuité est atteint, il y a un sens à dire que nous avons affaire à une forme indivisible, c'est-à-dire à un être qui ne se pense qu'à partir de cette unité :

Presque toutes les formes de la matière sont indivisibles. Il n'y a pas la moitié d'un doigt, la moitié d'un œil; il n'y a pas la moitié d'une molécule quelconque, parce que l'une des moitiés occupant une autre place que l'autre moitié, par cette seule et unique raison, elle ne lui ressemble pas (Diderot, Observations sur Hemsterhuis, DPV, XXIV, p. 297) ${ }^{25}$.

L'indivisibilité des «formes» ainsi envisagée illustre qu'en dépit de leur caractère transitoire, il existe des parties de matière qui, au sein même du continuum

23 Comment ne pas évoquer ici l'article fameux de l'Encyclopédie (X, p. 602) signé de la main de Diderot - Modification, Modifier, Modificatif, Modifiable: «Il n'y a pas un atome dans la nature qui ne soit exposé à l'action d'une infinité de causes diverses; il n'y a pas une de ces causes qui s'exercent de la même manière en deux points différents de l'espace: il n'y a donc pas deux atomes rigoureusement semblables dans la nature».

24 Pour d'autres développements sur cette question, voir Cherni (2002, p. 231 et sq.).

25 Comparez avec Le rêve de d'Alembert (DPV, XVII, p. 106-107) : «Est-ce que vous ne voyez pas que toutes les qualités, toutes les formes sensibles dont la matière est revêtue sont essentiellement indivisibles? Il n'y a ni plus ni moins d'impénétrabilité. Il y a la moitié d'un corps rond, mais il n'y a pas la moitié de la rondeur; il y a plus ou moins de mouvement, mais il n'y a ni plus ni moins mouvement; il n'y a ni la moitié, ni le tiers, ni le quart d'une tête, d'une oreille, d'un doigt, pas plus que la moitié, le tiers, le quart d'une pensée. Si dans l'univers il n'y pas une molécule qui ressemble à une autre, dans une molécule pas un point qui ressemble à un autre point, convenez que l'atome même est doué d'une qualité, d'une forme indivisible; convenez que la division est incompatible avec les essences des formes, puisqu'elle les détruit.» 
1 de la nature, se comportent comme des unités discrètes parce que leur mode de maintient tel tant que rien ne vient rompre cette relation : «Vivant, j'agis et je réagis en masse... mort, j'agis et je réagis en molécules», dit Le rêve de d'Alembert (DPV, XVII, p. 139). Il n'est évidemment pas nécessaire que l'unité de l'animal soit chaque fois correspondante à ce qui est perçu tel : certains animaux débordent notre échelle de perception parce qu'ils sont trop petits; de la même manière, il peut exister des super-organismes que nous voyons comme des parties détachées parce que ce qui les unit ne nous est pas perceptible. Autrement dit, l'échelle de ce qui constitue une forme indivisible n'est pas assignable à l'ordre des phénomènes perçus, et conséquemment il est impossible de fixer a priori où commencent et où finissent les individus qui peuplent l'univers.

\section{La question de l'identité}

L'idée, évoquée dans la dernière citation, que «les formes de la matière sont indivisibles» permet de faire le passage de la question de l'individuation à celle de l'identité. En effet, la théorie de l'individuation se veut une explication du mode de constitution de ces masses partielles qui deviennent des formes indivisibles, des parties de matière agissant «en masse» comme un «animal un», c'està-dire comme une entité qui, dans ses actions et réactions, au fil des accidents qui forment la trame de son existence, préserve une certaine identité à soi.

Dans un système de type cartésien, ces deux questions sont en quelque sorte séparées parce que les corps, étant conçus comme des parties de matière se mouvant ensemble, n'ont pas en eux-mêmes la raison de leur identité dans le temps - celle-ci est purement phénoménale. En réalité, seule la substance matérielle dans son ensemble constitue un individu «corporel». Conséquemment, chaque substance est un individu à part entière, ce qui signifie que l'identité humaine, l'identité du moi, est seulement attribuée aux substances pensantes aux âmes. Dans ce contexte, le fait que le corps auquel chaque âme est unie soit en perpétuel changement ne pose aucun problème : il entre dans la question de l'individuation des corps, sans être l'objet de la théorie de l'identité.

La critique lockéenne de la conception cartésienne du sujet comme substance pensante - fondée sur l'idée que penser n'est qu'une des fonctions de l'âme, non son essence - a conduit à repenser la catégorie d'identité à soi en la fondant sur les notions de conscience et de mémoire ${ }^{26}$. Seulement, cette critique de Locke ne change rien à ce qui constitue l'enjeu véritable de l'effort de Diderot pour produire une conception matérialiste cohérente de l'identité,

26 On lira à ce sujet les remarques très intéressantes sur l'évolution des usages du mot «conscience» depuis Locke et sa traduction en français par Coste, qui le colore de malebranchisme, jusqu'aux philosophes des Lumières françaises à partir des années 1740, dans Davies (1990, p. 70-95). 
à savoir le fait que, d'une part, l'identité à soi ressentie sous la forme du moi semble tenir à la conscience, donc à une forme d'intériorité inaccessible à autrui ${ }^{27}$, et que, d'autre part, ce qui lui donne une existence comme chose identique à elle-même pour autrui tient au fait qu'elle est attribuable à un corps en constant changement. Locke, même s'il s'est débarrassé de la notion de substance pour fonder la réalité du moi, rapporte l'identité personnelle à la conscience où les sensations qui sont actuelles se lient à celles qui sont dans la mémoire et forment une continuité dans le temps indépendante de son substrat matériel ${ }^{28}$.

La difficulté des positions de Descartes et de Locke réside dans le fait que l'identité personnelle, pour n'être pas fondée dans le corps, est rapportée au champ de la conscience : pour Descartes, cela oblige à accepter que l'âme pense toujours, puisque c'est son essence; pour Locke, cela conduit à traiter la vie non consciente comme hors du champ de l'identité personnelle. Dans les deux cas, donc, il n'y a d'identité à soi que du côté de notre part immatérielle, en tant qu'elle est conscience de soi. C'est cette primauté de la perspective en première personne qui constitue le noyau du problème. Peut-être, en effet, faut-il admettre que ce que je suis - et donc ce que je peux devenir ou avoir été - déborde le savoir que j'ai à mon sujet ${ }^{29}$.

Le moi est le résultat de la mémoire qui attache à un individu, la suite de ses sensations. Si je suis un individu, c'est moi. Si c'est un autre individu, c'est lui. Le lui et le moi naissent du même principe (Diderot, Observations sur Hemsterhuis, DPV, XXIV, p. 329).

Le soi d'un autre, ou le lui d'un jeune homme, d'un fils, par exemple, peut être plus étendu pour un père que pour son enfant (Diderot, Observations sur Hemsterhuis, DPV, XXIV, p. 303).

27 Ce que la critique contemporaine appelle parfois la spécificité de la perspective en première personne (Wolfe, 2011, p. 145 et sq.).

28 «Nous pouvons voir par-là en quoi consiste l'identité personnelle; \& qu'elle ne consiste pas dans l'identité de Substance, mais comme j'ai dit, dans l'identité de con-science» (Locke, 1735, II, §19, p. 271).

29 C. Jacot-Grapa (2009, p. 378-382) fait remarquer que l'une des difficultés rencontrées par la théorie lockéenne de l'identité est que les épisodes de la vie comme le rêve sont pour ainsi dire évacués de ce qui fait être une personne ce qu'elle est en effet, puisque dans le rêve, le moi n'est pour ainsi dire pas lui-même - pour Diderot, au contraire, l'aliénation fait partie de la vie du sujet. Martin Lin (2005, p. 243-268) fait le même constat dans un effort pour montrer que la philosophie spinoziste, parce qu'elle prend en compte l'individualité physique, parvient à surmonter les apories de Locke. Bien qu'il n'y ait pas de continuité historique démontrable de Spinoza à Diderot sur cette question, on voit que leurs monismes respectifs les rapprochent quant à la démarche. 


\subsection{Le lui que je suis}

De fait, il est peut-être nécessaire de traiter l'identité personnelle consciente comme un dérivé d'un processus d'individuation ayant lieu en amont. C'est, du moins, ce que Diderot semble proposer. Pour ce faire, il commence donc par rendre compte du mode de cohésion des parties de matière dont un corps est le résultat, en montrant qu'il «agit en masse», c'est-à-dire comme un individu indépendamment du fait qu'il soit doué de conscience — de manière à rendre compte, donc, du lui que je suis sans faire référence au moi. L'idée, au fond, est de montrer que «l'animal un», même s'il est emporté dans le mouvement général qui affecte le tout — seul véritable individu — constitue une unité assez stable pour former un type d'individu. À ce titre, s'il est vrai que l'identité personnelle tient à l'histoire qui attache à cet individu une suite de modifications, rien n'empêche de faire commencer cette histoire avant qu'il n'y ait conscience.

L'idée que la sensibilité soit une propriété incompatible avec l'essence de la matière est l'obstacle métaphysique par excellence sur ce point. En posant en principe, comme c'est le cas en général à l'âge classique, que la sensibilité est une propriété des esprits, on est reconduit à une ambiguïté : tout ce qui apparaît comme un individu doué d'une certaine identité n'est de fait tel que si on peut aussi lui prêter un esprit dont le corps n'est que le signe — puisque lui-même n'est un individu que dans le champ phénoménal de celui qui le perçoit. Autrement dit, le corps ne peut pas être le sujet de son histoire. Il forme un tout pour l'esprit qui le perçoit et pour celui qui lui est uni mais, dans les deux cas, cela est un pur effet de la perception confuse de parties de matière se mouvant ensemble. L'unité du corps matériel a donc son fondement hors de lui dans les deux cas, c'est-à-dire dans une conscience dont l'existence est ainsi posée de manière séparée.

Pour Diderot, il est philosophiquement plus économique d'accepter l'hypothèse que la sensibilité puisse être une propriété de la matière, propriété dont le degré d'activité, pour chaque partie, varie en fonction des rapports que cette partie entretient avec son environnement ${ }^{30}$. Sensibilité, dans ce contexte,

30 Cette hypothèse est discutée, dans Le rêve de d'Alembert, sous la forme d'une thèse d'apparence paradoxale que l'on doit simplement s'efforcer de rendre plausible : «D'Alembert- - [...] cette sensibilité que vous lui substituez [à l'hypothèse de l'existence d'un être non matériel], si c'est une qualité générale et essentielle de la matière, il faut que la pierre sente. DIDEROT — Pourquoi non? [...] On fait du marbre avec de la chair, et de la chair avec du marbre [...] D'Alembert — Serait-ce par hasard que vous reconnaîtriez [...] une sensibilité active qui se caractérise par certaines actions remarquables dans l'animal et peut-être dans la plante; et une sensibilité inerte dont on serait assuré par le passage à l'état de sensibilité active. [...] Je ne vois pas trop comment on fait passer un corps de l'état de sensibilité inerte à l'état de sensibilité active. Diderot — Cela se fait toutes les fois que vous mangez [...] car en mangeant que faites-vous? Vous levez les obstacles qui s'opposaient à la sensibilité active de l'aliment...» (DPV, XVII, p. 89-93). 
désigne avant tout une capacité d'être le sujet d'une modification, mais aussi de réagir à cette modification ${ }^{31}$. Chaque partie de matière est alors le sujet de sa propre histoire, chaque partie de matière est un individu, puisque chaque partie de matière est en elle-même le sujet de modifications.

Or, ce n'est pas tout : la propriété de la matière de pouvoir adopter des modes de cohésion qui font passer d'un état de contiguité à un état de continuité a son pendant dans la sensibilité : deux parties sensibles de matière, qui sont chacune le sujet unique de leur histoire propre, lorsque liées de manière à former un «animal un» agissant et réagissant «en masse», deviennent le sujet commun d'une histoire commune ${ }^{32}$. Ainsi, les molécules s'agglomèrent pour former des fibres, ces fibres s'agencent entre elles pour constituer des faisceaux, puis des organes qui, eux-mêmes, en viennent à se grouper en un corps complexe. Si bien que le lui que je suis, l'individu que je suis, ce qui est constitutif de mon identité personnelle n'est pas réductible à la conscience ou à la mémoire consciente. De fait, toutes les modifications qui affectent mon corps lui donnent son histoire propre, histoire dont les épisodes conscients ne forment qu'une partie. Un être extérieur qui se placerait au bon endroit pourrait suivre le développement de cette identité depuis un temps qui précède toute forme de conscience.

BoRDEu - D'abord vous n'étiez rien. Vous fûtes, en commençant, un point imperceptible, formé de molécules plus petites éparses dans le sang, la lymphe de votre père ou de votre mère; ce point devint un fil délié, puis un faisceau de fils. [...] Chacun des brins du faisceau de fils se transforma, par la seule nutrition et par sa conformation, en un organe particulier. [...] le faisceau est un système purement sensible [...]. Mais cette sensibilité pure et simple, ce toucher se diversifie par les organes émanés de chacun des brins; un brin formant une oreille, donne naissance à une espèce de toucher que nous appelons bruit ou son; un autre formant le palais, donne naissance à une seconde espèce de toucher que nous avons appelons saveur (Diderot, Le rêve de d'Alembert, DPV, XVII, p. 118).

On a ainsi un système d'échelles : il y a la vie à l'échelle de la molécule, du brin, du faisceau, de l'organe et du corps. À chacun de ces degrés correspond

31 Pour plus de détails sur cette notion, voir Hisayasu Nakagawa (1999, p. 199-218).

32 Diderot présente les choses ainsi : «Et la formation de cette continuité? Elle ne l'embarrassera guère... Comme une goutte de mercure se fond dans une autre goutte de mercure, une molécule sensible et vivante se fond dans une molécule sensible et vivante... D'abord, il y avait deux gouttes, après le contact il n'y en a plus qu'une... Avant l'assimilation il y avait deux molécules, après l'assimilation il n'y en a plus qu'une... La sensibilité devient commune à la masse commune... En effet, pourquoi non?... Je distinguerai par la pensée sur la longueur de la fibre animale tant de parties qu'il me plaira, mais la fibre sera continue, une» (Le rêve de d'Alembert, DPV, XVII, p. 117). 
1 un type «d'animalité» spécifique, c'est-à-dire une manière pour l'individu 2 formé d'agir et de réagir «en masse». En ce sens, le lui que je suis pour 3 quelqu'un d'autre a commencé bien avant que je ne sois un moi pour moi-

33 Il n'y a pas, autrement dit, de «monade dominante» comme on en trouve chez Leibniz, où l'identité du sujet à travers le temps est assurée.

Quand je naquis, écrit Diderot, je ne sentais que sur une longueur d'environ dix-huit pouces au plus. Comment suis-je parvenu avec l'âge à sentir sur une longueur de cinq pieds et quelques pouces? J'ai mangé. J'ai digéré. J'ai animalisé. J'ai fait passer par l'assimilation, des corps bruts, de l'état de sensibilité inerte à l'état de sensibilité active (Diderot, Observations sur Hemsterhuis, DPV, XXIV, p. 304).

La spécificité du mode d'individuation des corps complexes comme ceux des animaux est qu'ils sont constitués d'une multitude d'organes ayant chacun leur fonction propre. C'est ce qui fait que l'animal comme tout est susceptible d'être affecté de plusieurs sensations simultanées et d'en faire une sorte de synthèse : ce qui est senti par l'œil, l'oreille et la main dans un même instant met en branle le système nerveux de sorte que le corps entier produit une seule développe par agglomération de molécules. À ce stade, ce que je suis a commencé d'être, même s'il n'y a pas de conscience (ou de mémorisation consciente) au sens propre. Un sujet est en train de se développer, mais il n'est pas localisé dans telle ou telle partie de l'animal ${ }^{33}$, ni uni à lui comme s'il lui était extérieur. Il se forge de manière épigénétique par ce qu'il assimile et par sa manière de l'assimiler. À chaque moment de son existence, son organisation se déploie, se modifie au gré de rencontres avec son environnement. Autrement dit : il est déjà un lui pour d'autres, alors qu'il n'est pas un moi pour lui-même. est déjà, donc, un individu, dont l'identité à soi dans le temps est assurée par

Au cœur de l'argument se trouve le fait que le lui que je suis existe à titre de sujet parce qu'un autre que moi peut lui rattacher une suite de sensations - en d'autres termes, le processus d'individuation physique qui fonde l'identité à soi du sujet est attesté par la mémoire d'une autre personne. Ce que je suis, ce que je deviens inclut donc ces événements qui se produisent hors du champ de ma conscience, et qui en assurent la constitution singulière. Mémoire et conscience ne sont que certains des développements possibles et transitoires de l'histoire de ce sujet — dussent-elles n'être jamais formées, il y aurait eu processus d'individuation et vie animale tout de même. Il y a donc identité à soi d'un sujet à travers le temps, puisque chaque fois il agit ou réagit «en masse», faisant ainsi qu'une suite d'actions et de réactions puissent être rattachées à un même individu. 
1 réaction qui résulte de l'ensemble de ces affections. Chaque organe a une 2 réaction propre à l'affect pour lequel il est configuré mais, par rapport à l'animal 3 entier, il fait à la fois office 1) d'agent causal en tant qu'il modifie le corps à 4 partir de l'affect qu'il a pour fonction de recevoir et 2) d'entité passive en tant 5 qu'il est modifié par le système nerveux par où transigent toutes les affections 6 du corps. L'unité de l'animal ne tient donc pas au fait qu'il y ait un centre - le partie peut subir des modifications sans qu'il n'y ait de rupture dans le système général. Tout est affaire d'intégration : des parties de matière se détachent de l'individu, d'autres se greffent à lui, mais l'identité générale se maintient par la capacité de cette organisation à préserver son unité. Les molécules qui forment un organe sont échangées avec l'environnement sans que la vie de l'organe ne soit en danger; certains organes peuvent être séparés du corps sans que sa vie comme corps ne soit en danger - d'autres sont cruciaux pour le maintien de l'organisation, mais n'en sont pas pour autant le lieu où le moi se tient.

Il y a certainement dans un même animal trois vies distinctes. La vie de l'animal entier. La vie de chacun des organes. La vie de la molécule ou de l'élément. L'animal entier vit privé de plusieurs de ses parties. Le cœur, les poumons, la tête, la main, presque toutes les parties de l'animal entier vivent un temps considérable séparées du tout. Il n'y a que la vie de la molécule ou sa sensibilité qui ne cesse point; c'est une de ses qualités aussi essentielles que son impénétrabilité. La mort s'arrête là (Diderot, Fragments dont on n'a pu retrouver la place, DPV, XVII, p. 226).

En fait, la question de l'identité à soi de l'individu est entièrement ramenée par Diderot au domaine de la temporalité : est identique à soi une organisation dont les modifications ne sont pas suffisantes pour causer une rupture de son économie générale - c'est donc la perpétuation dans le temps d'un certain nombre de rapports qui compte ici. L'image qui décrit le mieux ce principe pour Diderot est celle du monastère :

BORDEU - Si vous eussiez passé en un clin d'œil de la jeunesse à la décrépitude, vous auriez été jeté dans ce monde comme au premier moment de votre naissance; vous n'auriez plus été vous ni pour les autres ni pour vous, pour les autres qui n'auraient point été eux pour vous. Tous les rapports auraient été anéantis, toute l'histoire de notre vie pour moi, toute l'histoire de la mienne pour vous, brouillée. Comment auriez-vous pu savoir que cet homme, courbé sur un bâton, dont les yeux s'étaient éteints, qui se traînait avec peine, plus différent encore de lui-même au dedans qu'à l'extérieur, était le même qui la veille marchait si légèrement, remuait des fardeaux assez lourds, pouvait se livrer aux méditations les plus profondes, aux exercices les plus doux et les plus violents? Vous n'eussiez pas entendu vos propres ouvrages, vous ne vous fussiez pas reconnu vous-même, vous n'eussiez reconnu personne, personne ne vous eût reconnu; toute la scène du monde aurait changé. Songez qu'il y eut moins de différence encore entre vous naissant et vous jeune, qu'il n'y en aurait 
entre vous jeune et vous devenu subitement décrépit. Songez que, quoique votre naissance ait été liée à votre jeunesse par une suite de sensations ininterrompues, les trois premières années de votre existence n'ont jamais été l'histoire de votre vie. D'Alembert décrépit n'eût pas eu le moindre souvenir de d'Alembert jeune [...].

$M_{A D}{ }^{L L E}$ DE L'ESPINASSE - Je dis que l'esprit monastique se conserve parce que le monastère se refait peu à peu, et quand il entre un moine nouveau, il en trouve une centaine de vieux qui l'entraînent à penser et à sentir comme eux. Une abeille s'en va, il en succède dans la grappe une autre qui se met bientôt au courant (Diderot, Le rêve de d'Alembert, DPV, XVII, p. 164-165).

Ce qui frappe dans cette description de l'identité, c'est peut-être le fait qu'elle inclut la possibilité d'une différentiation à soi absolument radicale : d'Alembert jeune et d'Alembert vieux n'ont aucune propriété interne ou externe qui permettrait de statuer sur leur identité. Il n'y a d'identité que s'il y a persistance de rapports dans le temps - mais de rapports qui peuvent exister sans mémoire. Ainsi, l'unité de l'animal humain vient de ce que les divers organes dont il est constitué voient leurs modifications mises en rapport de manière à coordonner une réaction «en masse» de l'ensemble de l'organisation. Inspiré de la médecine de Montpellier ${ }^{34}$, Diderot part du principe que l'organisation animale parvenue à un certain niveau de complexité est une structure où se coordonnent des mouvements animaux partiellement autonomes - le corps humain, en fait, est lui-même une multiplicité :

\section{$\mathrm{MAD}^{\mathrm{LLE}}$ DE L'EsPINASSE — Tous nos organes! [...]}

BORDEU - Après? Ne sont que des animaux distincts que la loi de continuité tient dans une sympathie, une unité, une identité générale (Diderot, Le rêve de d'Alembert, DPV, XVII, p. 122).

Autrement dit, le passage de contiguïté à continuité qui assure l'unité de l'animal n'interdit pas aux parties constitutives de cette unité de préserver une certaine autonomie (chaque organe agit en fonction de sa constitution propre) - ce qui conduit Colas Duflo à caractériser l'identité personnelle chez Diderot à partir de la notion de «moi-multiple». C'est que le moi est, dans tous les sens du terme, un rapport : rapport d'impressions diverses actuelles provenant de diverses parties de l'organisme, rapport d'impressions présentes à des impressions passées, etc. L'unité de l'animal n'est jamais que l'expression d'un certain degré d'intégration d'une multiplicité qui n'a pas de centre originaire (Duflo, 2008, p. 99). Évidemment, pour qu'il y ait rapport, il faut un organe qui serve en quelque sorte de lieu de rencontre; cet organe est bien évidemment le cerveau. Cependant, comme le remarque encore Colas Duflo,

34 Sur cette influence, voir, par exemple Arthur M. Wilson (1985, p. 469 et sq.). 
[c]et organe, pour être central, n'est jamais qu'un organe au sein d'une multiplicité d'organes qui nous constitue tout autant. En d'autres termes, mon cerveau, ce n'est pas moi. Il ne saurait à lui seul produire l'unité de l'ensemble dont il est une partie, et il est lui-même de surcroît constitué d'une multiplicité d'atomes sensibles (Duflo, 2008, p. 99).

Pour Diderot, en effet, le cerveau n'est qu'un organe fonctionnel assurant la liaison sensible entre les parties : un organe dont la sensibilité est tournée vers les autres organes du corps, comme une araignée dans sa toile, placée au milieu de son faisceau de fils tendus dans toutes les directions, qui sent chacune des modifications dans sa toile et peut les localiser. De telle sorte, l'identité personnelle est ramenée à sa plus simple expression : il s'agit d'une économie qui permet à une organisation de se maintenir dans un état de cohésion au travers du temps, mais dont les vicissitudes incessantes, à terme, finissent par ruiner l'équilibre.

\subsection{Le moi que je suis}

On pourrait dire, à l'instar de Franck Salaün (2010, p. 81-82), que le moi est un complexe de rapports au sein de l'individu et entre l'individu et ce qui lui est extérieur. Par là se voit réintroduite une perspective au départ mise de côté : celle du moi entendu comme conscience de soi. Sa mise de côté visait à montrer que Diderot procède en regardant le phénomène de l'identité d'abord selon une perspective en troisième personne : l'identité du soi d'un autre déborde le phénomène de sa conscience, et donc de l'histoire consciente de sa vie. Du point de vue purement physiologique, cela signifie simplement qu'il n'y aura conscience que lorsque les conditions développementales du cerveau auront été atteintes. Philosophiquement, cela implique que la conscience de soi est simplement comprise comme une fonction d'un certain état de l'organisation - Diderot corporalise l'argument de Locke qui faisait de la pensée une fonction de l'âme...

En d'autres termes, la conscience doit être conçue comme l'effet d'une métamorphose de l'organisation des parties de matière qui forment le corps. La 39 matière — dont on sait qu'elle est sensible — se voit ainsi prêter une capacité d'acquérir des propriétés au fil de ses métamorphoses.

Vous avez été un atome de ce grand tout, le temps vous réduira à un atome de ce grand tout. Chemin faisant, vous aurez passé par une multitude de métamorphoses. De ces métamorphoses, la plus importante est celle sous laquelle vous marchez à deux pieds, la seule qui soit accompagnée de conscience, la seule sous laquelle vous constituez par la mémoire de vos actions successives, un individu qui s'appelle moi (Diderot, «Compte rendu de Dieu et l'homme de M. de Valmire», DPV, XX, p. 655-656. Ce texte date de la période 1771-1773). la pensée, au raisonnement, sans autre agent ou intermède que des agents ou inter44 mèdes matériels (Diderot, Observations sur Hemsterhuis, DPV, XXIV, p. 277). 
1 Comme ce passage n'est qu'affaire d'organisation, à partir du moment où la

2 sensibilité est active, on peut dire qu'il existe une forme de conscience -

3 fût-ce à un stade embryonnaire. À chaque état d'organisation correspond une

4 manière d'être conscient de son existence en tant qu'animal un, puisqu'il y a

5 réaction «en masse» à un affect qui, lui, peut très bien être local. Qu'il s'agisse

6 d'un polype ou d'un grand mammifère, la différence n'est que de mode

7 d'organisation, et donc de manière dont cette conscience se manifeste et dé-

8 ploie ses effets. Dans le cas des animaux doués de mémoire, un organe s'est

9 développé, le cerveau, dont la fonction est définie à partir d'un modèle

10 centre-périphérie par Diderot : les organes ont diverses sensations qu'elles rap-

11 portent vers un organe central, lequel localise leur provenance et coordonne

12 une réponse de l'ensemble de l'organisme par une action en retour. Ainsi, les

13 diverses sensations qui affectent le corps en un instant donné sont à la fois

14 mises en rapport entre elles et mises en rapport avec des sensations passées,

15 hiérarchisées et ordonnées. Il y a donc unité de l'animal au sens où il y a liaison

16 de sensations diverses et rattachement à un individu ${ }^{35}$.

De ce point de vue, le cerveau apparaît comme un point d'ancrage pour une sorte de sens interne, lequel désigne une voie d'accès épistémologique particulière au corps propre (Wolfe, 2011, p. 146-147). La conscience de soi est littéralement une connaissance de ce qu'une modification qui affecte tel organe m'affecte moi comme individu, et de ce que la sensation de cette modification est liée à celles qui ont précédé, formant la trame de mon histoire consciente. De là s'ensuit qu'il faut admettre que le lui que je suis pour quelqu'un d'autre et le moi que je suis pour moi-même ne coïncident pas. Cette non-coïncidence va en quelque sorte de soi dès lors que l'on accepte qu'il y a décalage entre l'individualité corporelle et l'identité psychologique. C'est qu'en effet, l'histoire de la vie de mon corps pour quelqu'un d'autre que moi n'est pas la même que pour moi-même, et, qui plus est, aucune de ces histoires n'est complète, puisque cette vie commence dans la vie des molécules dont il est constitué, dont personne n'a de perception consciente. En définitive, le moi que je suis outrepasse de toutes parts les histoires qu'on en fait. L'idée que nous nous faisons de nousmêmes et d'autrui est toujours partielle et limitée.

Je voudrais terminer ce parcours par trois remarques sur ce décalage, afin de montrer comment Diderot problématise toute tentative de réduction du moi à la conscience, et conteste ce faisant toute simplification des notions de responsabilité morale et juridique, d'authenticité, de maîtrise ou de connaissance de soi. Les exemples invoqués par Diderot dans cette démarche sont

35 Jean-Claude Bourdin écrit : «On peut alors poser que c'est dans le rapport qui s’effectue naturellement, par habitude et expérience accumulée, entre les sensations et le centre commun que réside “l'unité de l'animal”» (1999, p. 63). 
1 des cas pathologiques ${ }^{36}$ - mais ceux-ci ne constituent en fait que des pointes extrêmes qui illustrent merveilleusement ce qui est en cause.

1) La thèse suivant laquelle la conscience de soi est un effet du raccordement d'organes aux fonctions relativement autonomes autour d'un système nerveux qui permet au cerveau d'être affecté par leurs mouvements respectifs amène Diderot à souligner le fait qu'il existe des perturbations du rapport entre le corps réel et le corps senti (Bourdin, 1999, p. 65) : le corps réel est affecté par l'extérieur, mais toutes ces affections ne font pas l'objet d'une sensation actuelle. C'est que les rapports qui s'établissent entre les parties du corps sont eux-mêmes variables, de sorte que la conscience est plus ou moins mobilisée par telle ou telle partie au détriment du reste. Diderot aborde souvent cette question sous l'angle d'un rapport non hiérarchisé entre centre et périphérie : il arrive que le cerveau domine, et alors il est moins soumis aux aléas des affects du corps; d'autres fois, la «sensibilité» domine, et alors le cerveau se laisse entrainer par le corps. Mais une telle description doit être complétée : la périphérie en général n'existe pas, il faut ajouter au tableau le fait que c'est tantôt tel ou tel organe qui peut dominer le système, de sorte que le rapport centrepériphérie est en fait un rapport entre des organes divers où tout excès de domination d'un organe sur le système est considéré comme mettant en danger l'organisation. On comprend alors que ce soit dans une sorte d'équilibre de ce système que la relation du moi conscient au corps propre est considérée comme adéquate. Les variations de cet équilibre constituent la trame des états qui caractérise la vie individuelle, et la gravité des écarts par rapport à l'équilibre permet d'envisager des pathologies du moi.

2) La conscience de soi survenant graduellement au cours du développement du corps, elle apparaît déjà singularisée par une histoire à laquelle elle ne peut avoir accès par la mémoire, et qui pourtant la détermine. À ce titre, les passions, désirs, envies, etc. peuvent avoir une origine dans une sorte de préhistoire du moi, et être au moins pour partie hors de contrôle. Mais ce qui est ici vrai pour son apparition vaut en fait pour toute la vie : il est possible qu'une condition du corps occasionne des ruptures dans l'histoire de vie consciente de l'individu. Suivant une perspective qui réduit le moi à la mémoire et à la conscience, ces épisodes doivent être

36 Dans Le rêve de d'Alembert, Diderot convoque un grand nombre de cas particuliers à titre d'expériences de pensée : prêtre enthousiaste qui parvient à s'exempter de la souffrance d'une opération à froid par la prière, siamoises ayant des périodes de conscience en alternance, philosophe se plongeant dans un problème de métaphysique pour oublier un mal d'oreilles, cas d'amnésie totale, de léthargie incurable, de trépanation, etc. 
considérés comme des moments où l'on est «hors de soi». Pourtant, si l'on tient compte du fait, évoqué plus tôt, qu'il est possible que le lui d'un enfant soit plus étendu pour son père que pour lui-même, cela signifie proprement que, pour Diderot, ces épisodes, même ignorés, font partie intégrante de l'identité de l'individu. D'où la présence de ce que Jacques Chouillet (1984, p. 214-225) identifie comme une double hypothèse sur la mémoire qui parcourt l'œuvre de Diderot : une mémoire «totale» représente le fait que chaque modification du corps est pour ainsi dire enregistrée en lui et y demeure puisque, l'ayant modifié, elle participe à ce qu'il est; et une mémoire partielle incarne l'idée consciente que la personne a d'elle-même, ou la série des sensations actuellement présentes dans la conscience que le moi a de lui-même. Cette double caractérisation rend compte du fait que le moi est une idée partielle de ce qu'est l'individu, et qu'en deçà de cette idée, il peut s'accumuler des modifications capables d'induire des comportements inattendus ou des effets de résonnance par association d'idées réveillant des souvenirs enfouis. Diderot écrit à ce titre :

\begin{abstract}
Ainsi, la mémoire immense, c'est la liaison de tout ce qu'on a été dans un instant à tout ce qu'on a été dans le moment suivant; états qui, liés par l'acte, rappelleront à un homme tout ce qu'il a été toute sa vie.

Or, je prétends que tout homme a cette mémoire. [...]

La mémoire immense ou totale est un état d'unité complet; la mémoire partielle, état d'unité incomplet (Diderot, Éléments de physiologie, dans 1875, IX, p. 370).
\end{abstract}

3) Cet écart entre la mémoire totale et la mémoire partielle permet par ailleurs de rendre compte du fait plus général que ce qui nous fait agir déborde souvent le cadre de la conscience et, conséquemment, met en évidence le décalage épistémique entre identité personnelle et individualité. Comme le dit Jacques Chouillet, «[u]ne fois pour toutes doit être écarté, pour une lecture correcte de Diderot, le schéma grammatical où la place du sujet est donnée à l'âme, celle du verbe à une action de l'âme...» (1984, p. 214). De fait, l'image que nous nous faisons de nous-mêmes, parce qu'elle tient à ce qui est actuellement présent à la conscience, qui comprend les sensations les plus prégnantes et celles qu'elles font ressurgir par association, varie au fil des événements qui forment la trame de notre vie. Dans le cours normal de la vie, nous agissons au gré des habitudes que nous avons contractées et ignorons tout ce dont notre corps est capable, et qui pourra être réveillé subitement. Ainsi :

L'animal tranquille ignore sa force. Mais qu'un accès de fièvre, un transport de passion, un violent intérêt, un mouvement d'amour-propre, la crainte de perdre son honneur, sa vie ou sa fortune, une terreur réelle ou panique, tende 
ses nerfs, accélère l'impulsion de ses fluides, resserre les canaux qui les referment, et surtout que la velléité, volonté ou âme ne s'en mêle plus, et vous serez effrayé des effets qu'il produira (Diderot, Observations sur Hemsterhuis, DPV, XXIV, p. 305-306).

\section{Conclusion}

On comprend par ces dernières remarques que Diderot s'efforce de donner aux décalages entre individuation corporelle et identité psychologique une explication qui ne tienne pas à la dualité des substances. L'identité personnelle, de fait, n'est pas réductible à la conscience, mais trouve plutôt son foyer dans le corps; en revanche, tous les corps qui font partie de l'ordre phénoménal ne peuvent pas être considérés comme doués d'une identité personnelle au sens propre, puisque seuls certains d'entre eux agissent et réagissent véritablement «en masse». La condition réelle pour que l'on puisse parler d'identité à soi d'un individu est qu'il y ait persistance dans le temps de certains rapports de manière à ce qu'un équilibre organisationnel soit maintenu. En ce sens, il est vrai que le moi est la suite des sensations que j'ai éprouvées et qui font l'histoire de ma vie, histoire qui commence un peu plus tôt ou un peu plus tard pour un individu et pour un autre individu. Le moi que je crois être et le lui que j'attribue aux autres relèvent tous deux d'un effort pour saisir l'unité d'un corps au sujet duquel nous n'avons qu'un savoir fragmentaire. Nous ne sommes jamais le même pour les gens qui nous entourent, puisque chaque personne a une suite différente de sensations qu'elle attache à mon individualité, mais cette différence a précisément son fondement dans le fait que mon existence est indépendante de la perception qu'on en a.

D'une certaine manière, le moi est une fiction, au sens où il s'agit d'une idée que nous nous faisons au sujet de nous-mêmes - tout comme le lui est une idée que nous nous faisons d'autrui. Les limites épistémologiques à la connaissance adéquate ou totale d'un individu singulier que nous avons évoquées tout au long de ce qui précède nous obligent à considérer ces idées incomplètes que nous nous faisons au sujet de nous-mêmes et des autres comme responsables pour partie de ces décalages. Il est de la plus haute importance de reconnaître ce décalage épistémique : en effet, c'est à partir de cette fiction que nous sommes pour nous-mêmes et que les autres sont pour nous que nous prenons des décisions sur ce que nous pouvons et devons faire. Nier ce décalage et croire que ce que nous sommes se réduit à l'idée que nous en avons entraine généralement, pense Diderot, des décisions mal éclairées. L'exemple typique est celui, très souvent évoqué, du mariage... dont Diderot écrit qu'il s'agit d'une

41 instant aveugle sur l'instabilité de tout ce qui les entoure (Diderot, Supplément au 44 voyage de Bougainville, dans 1875, II, p. 242). 
1 Au-delà des cas pathologiques de décalages entre les états du corps et ceux de

2 la conscience, Diderot évoque souvent ces conventions sociales fondées sur

3 l'idée que l'identité à soi d'une chose renvoie à une essence fixe et immuable

4 pouvant être saisie et avec laquelle un rapport authentique peut être engagé.

5 Admettre, au contraire, que le moi n'est jamais qu'une idée incomplète de

6 ce que nous sommes commande une authenticité d'un autre ordre : celle d'un 7 rapport sain avec le caractère instable des choses et de notre être même.

8 Le décalage entre identité psychologique et individuation physiologique

9 tient à ce que la conscience est traitée comme un pur effet de l'organisation

10 physique, et plus particulièrement comme une fonction de coordination exer-

11 cée par le cerveau. En ce sens, Diderot déplace le rapport entre substances qui

12 expliquait ce décalage le long d'un axe immanent à la matière : le moi surgit à

13 la faveur d'un certain rapport du cerveau au reste du corps, lui-même constitué

14 comme effet de rapports entre divers organes. Sa stabilité et son instabilité sont

15 donc à la mesure de la stabilité et de l'instabilité de toute chose - rien ne se

16 faisant par saut, mais tout étant voué à une vicissitude permanente.

17

Remerciements : Je tiens à remercier Éric Leduc et Maud Brunet-Fontaine qui m'ont assisté dans les recherches réalisées pour l'écriture de cet article, ainsi que les évaluateurs anonymes de Dialogue pour leurs commentaires qui m'ont aidé à parvenir à cette version définitive. Je tiens également à remercier Syliane Charles pour avoir organisé le symposium où j'ai présenté une version préliminaire de ce travail, ainsi que tous les participants à ce symposium qui, par leurs questions et commentaires, ont contribué à l'écriture de cet article.

\section{Références bibliographiques}

Audidière, Sophie

2006 «Individu», dans Sophie Audidière, Jean-Claude Bourdin et Colas Duflo, dir., L'Encyclopédie du Rêve de d'Alembert de Diderot, Paris, CNRS Éditions.

Baertschi, Bernard

2005 Conscience et réalité. Études sur la philosophie française au XVIII siècle, Genève, Droz (coll. «Bibliothèque des Lumières»).

Bourdin, Jean-Claude

1998 Diderot. Le matérialisme, Paris, Presses universitaires de France.

Bourdin, Jean-Claude

1999 «Les vicissitudes du moi dans Le Rêve de d'Alembert de Diderot», dans Jean-Noël Missa, dir., Matière pensante. Études historiques sur les conceptions matérialistes en philosophie de l'esprit, Paris, Vrin, p. 57-67.

Cammagre, Geneviève

2000 Roman et histoire de soi. La notion de sujet dans la Correspondance de Diderot, Paris, Champion. 
Cherni, Amor

2002 Diderot. L'ordre et le devenir, Genève, Droz.

Chouillet, Jacques

1984 «Matière et mémoire dans l'œuvre de Diderot», Revue de métaphysique et de morale, vol. 89, p. 214-225.

Davies, Catherine Glyn

1990 Conscience as Consciousness. The Idea of Self-Awareness in French Philosophical Writing from Descartes to Diderot, Oxford, Voltaire Foundation (coll. «Studies on Voltaire and the Eighteenth Century»).

Descartes, René

1897-1913 Euvres, sous la direction d'Adam et Tourneux, Paris, Cerf.

Diderot, Denis

1875 Euvres, édition par J. Assézat et M. Tourneux, Paris, Garnier.

Diderot, Denis

1975-2016 Euvres [DPV], sous la direction de H. Dieckmann, J. Proust et J. Varloot, Paris, Hermann.

Diderot, Denis et Jean le Rond d'Alembert, dir.

1751-1765 Encyclopédie ou Dictionnaire raisonné des sciences, des arts et des métiers, Paris, Durand et al.

Dion-Sigoda, Françoise

1992 «L'homme-clavecin : évolution d'une image», dans Jean-Louis Jam, dir., Éclectisme et cohérences des Lumières. Mélanges offerts à Jean Erhard, Paris, Nizet, p. 221-228.

Duchesneau, François

1999 «Diderot et la physiologie de la sensibilité», Dix-huitième siècle, vol. 31, p. 195-216.

Duflo, Colas

2006 Diderot philosophe, Pais, Champion.

Duflo, Colas

2008 «Le moi-multiple. Fondements physiologiques, conséquences anthropologiques», Archives de philosophie, vol. 71, n 1, p. 95-110.

Firode, Alain

2001 La dynamique de d'Alembert, Montréal/Paris, Bellarmin/Vrin.

Gandt, François de

2001 «Qu'est-ce qu'être newtonien en 1740?», Studies on Voltaire and the Eighteenth Century, no 11, p. 126-147.

Jacot-Grapa, Caroline

2009 Dans le vif du sujet. Diderot, corps et âme, Paris, Classiques Garnier.

Lin, Martin

2005 «Memory and Personal Identity in Spinoza», Canadian Journal of Philosophy, vol. 35, no 2, p. 243-268.

Locke, John

1735 Essai sur l'entendement humain, trad. Pierre Coste, Amsterdam, Pierre Mortier. 
Maizeaux, Pierre des

1720 Recueil de diverses pièces, sur la Philosophie, la Religion Naturelle, l'Histoire, les Mathématiques, \&, par Mrs. Leibniz, Clarke, Newton, et autres auteurs célèbres, I Tome, Amsterdam, Duvillard et Changuion.

Nakagawa, Hisayasu

1999 «Genèse d'une idée diderotienne : la sensibilité comme propriété générale de la matière», dans Béatrice Fink et Gerhardt Stenger, dir., Etre matérialiste à l'âge des Lumières. Hommage offert à Roland Desné, Paris, Presses universitaires de France, p. 199-218.

Pépin, François

2012 La philosophie expérimentale de Diderot et la chimie, Paris, Classiques Garnier.

Rioux-Beaulne, Mitia

2015 «"Laissez là vos individus": Diderot et Leibniz devant le bien-fondé des phénomènes», dans Christian Leduc, François Pépin, Anne-Lise Rey et Mitia Rioux-Beaulne, dir., Leibniz et Diderot. Rencontres et transformations, Montréal/Paris, Presses de l’Université de Montréal/ Vrin.

Rioux-Beaulne, Mitia

2016 «Des billevesées de l'une à la sublimité de l'autre : les métaphysiques de Diderot», dans Pierre Girard, Christian Leduc et Mitia Rioux-Beaulne, dir., Les Métaphysiques des Lumières, Paris, Classiques Garnier.

Salaün, Franck

1999 «L'identité personnelle selon Diderot», Recherches sur Diderot et l'Encyclopédie, no 26, p. 113-123.

Salaün, Franck

2010 Le genou de Jacques. Singularités et théorie du moi dans l'œuvre de Diderot, Paris, Hermann.

Sowaal, Alice

2004 «Cartesian Bodies», Canadian Journal of Philosophy, vol. 34, n², p. 217-240.

Spinoza, Baruch

1954 Éthique, trad. R. Caillois, Paris, Gallimard.

Wilson, Arthur M.

1985 Diderot : sa vie et son æuvre, trad. Gilles Chahine, Annette Lorenceau et Anne Villelaur, Paris, Robert Laffont/Ramsay.

Wolfe, Charles T.

2011 «Éléments pour une théorie matérialiste du moi», dans François Pépin, dir., La circulation entre les savoirs au siècle des Lumières. Hommage à Francine Markovits, Paris, Hermann. 


\section{AUTHOR QUERIES}

QA $\quad$ The distinction between surnames can be ambiguous, therefore to ensure accurate tagging for indexing purposes online (eg for PubMed entries), please check that the highlighted surnames have been correctly identified, that all names are in the correct order and spelt correctly. 